\title{
RF PLASMA SOURCE FOR HEAVY ION BEAM CHARGE NEUTRALIZATION
}

\author{
Philip C. Efthimion, Erik Gilson, Larry Grisham, Ronald C. Davidson, Plasma Physics Laboratory, \\ Princeton University, Princeton, NJ 08543 \\ Simon S. Yu and B. Grant Logan, Lawrence Berkeley National Laboratory, Berkeley, CA 94720
}

\begin{abstract}
Highly ionized plasmas are being used as a medium for charge neutralizing heavy ion beams in order to focus the ion beam to a small spot size. A radio frequency (RF) plasma source has been built at the Princeton Plasma Physics Laboratory (PPPL) in support of the joint Neutralized Transport Experiment (NTX) at the Lawrence Berkeley National Laboratory (LBNL) to study ion beam neutralization with plasma. The goal is to operate the source at pressures $\sim 10^{-5}$ Torr at full ionization. The initial operation of the source has been at pressures of $10^{-4}$ $-10^{-1}$ Torr and electron densities in the range of $10^{8}-10^{11}$ $\mathrm{cm}^{-3}$. Recently, pulsed operation of the source has enabled operation at pressures in the $10^{-6}$ Torr range with densities of $10^{11} \mathrm{~cm}^{-3}$. Near $100 \%$ ionization has been achieved. The source has been integrated with the NTX facility and experiments have begun.
\end{abstract}

\section{INTRODUCTION}

HIBALL-II is a possible heavy ion fusion reactor design [1]. The final focusing magnets must focus multiple heavy ion beams to a small spot size in the target chamber. This will require a deliberate charge neutralization of the ion beams. The plasma will be created or injected into the drift section between the last magnetic lens and the reactor chamber. The Neutralized Transport Experiment (NTX) [2] has been configured to investigate the most promising neutralization methods. One charge neutralization concept employs a heavy ion beam propagating through a highly ionized plasma column [3]. The cold plasma ion motion is neglected, and electrons from the cylindrical plasma move into the beam channel, reducing the net positive beam charge over the larger volume of the plasma channel. For NTX, ion beam densities are in the range of $10^{8}-10^{9} \mathrm{~cm}^{-3}$. Present calculations require the plasma to be in the range of 0.1-2 meters in length with an electron density comparable to 1100 times the beam density. The operating pressure for the plasma needs to be in the range of $10^{-6}-10^{-5}$ Torr to prevent neutrals from stripping the beam ions to higher charge states. Previous plasma sources tested operated continuously, but were limited to pressures greater than 0.5 mTorr. The heavy ion beams for fusion will have pulse lengths in the sub-microsecond range. Consequently, operating the plasma source in a pulsed mode has been investigated. Both the gas feed to the source and the RF power are pulsed to achieve near full ionization at pressures in the range of $10^{-6}-10^{-5}$ Torr during the first few milliseconds of source operation.
This operation results in plasma densities and operating pressures acceptable for charge neutralization experiments in NTX. The source has now been installed on NTX, and initial experiments have commenced. Here, the new pulsed source is described and its operating characteristics are compared to continuous sources previously investigated.

\section{CONTINUOUS ECR SOLENOID SOURCE}

The continuous plasma source is a simple electron cyclotron resonance (ECR) solenoid source. It is a 4" diameter cylinder and is approximately 12 " long. Simple 16-gauge wire is wound around the cylinder to create a solenoid field. Up to 15 amperes of current can be applied to the coil to create a 30 gauss field. A radio frequency (RF) antenna couples power into the cylinder through a quartz window mounted at one end of the cylinder. The antenna is a simple 3-turn spiral. The spiral antenna is cooled with water and mounted in an enclosure against the window to prevent wave leakage. The source operates at $13 \mathrm{MHz}$ frequency and at nominal power levels of $1 \mathrm{~kW}$. The spiral antenna is impedance matched to the $13 \mathrm{MHz}$ radio frequency source with a pair of tunable capacitors arranged in series and parallel to the antenna. The source operation near 0.5 mTorr exhibits an electron cyclotron resonance. When varying the field, the plasma density is a maximum when $\square \sim \square_{\mathrm{ce}}$ at 6.2 gauss, and indicates cyclotron wave damping. Here, $\square_{\mathrm{ce}}$ is the electron cyclotron frequency. The operation of this source behaves like similar sources studied in the literature. It has an operating range down to $0.5 \mathrm{mTorr}$. Below this pressure the source extinguishes.

\section{PULSED PLASMA SOURCE}

The pulsed plasma source (Fig. 1) has a 6-way cross at the center of its design. A turbo-pump attached to one face maintains the vacuum while the gas inlet and the RF quartz window are attached to the opposite face. A threeturn copper spiral antenna is situated inside a shielded box and faces the window. The RF matching network is directly connected to the antenna enclosure and is tuned to match the low impedance antenna to the $50 \mathrm{Ohm}$ transmission cable. Perpendicular to the turbo-pump and antenna, and directed into the page, are two flanges to the 6-way cross where the drift tube for the ion beam is connected. The plasma drifts into the center of the cross, and intersects the propagating ion beam. The source operates by applying a puff of argon gas and a pulse of $\mathrm{RF}$ power to the antenna. The potential advantages of 
pulsed operation are that it can easily operate at high peak RF power levels, and the amount of gas can be limited. The plasma density and the neutral gas pressure are issues primarily during the $100 \square \mathrm{s}$ the ion beam passes through the plasma. Consequently plasma parameters and neutral gas pressure are dynamic quantities and need to be measured as a function of time in order to evaluate source operation.

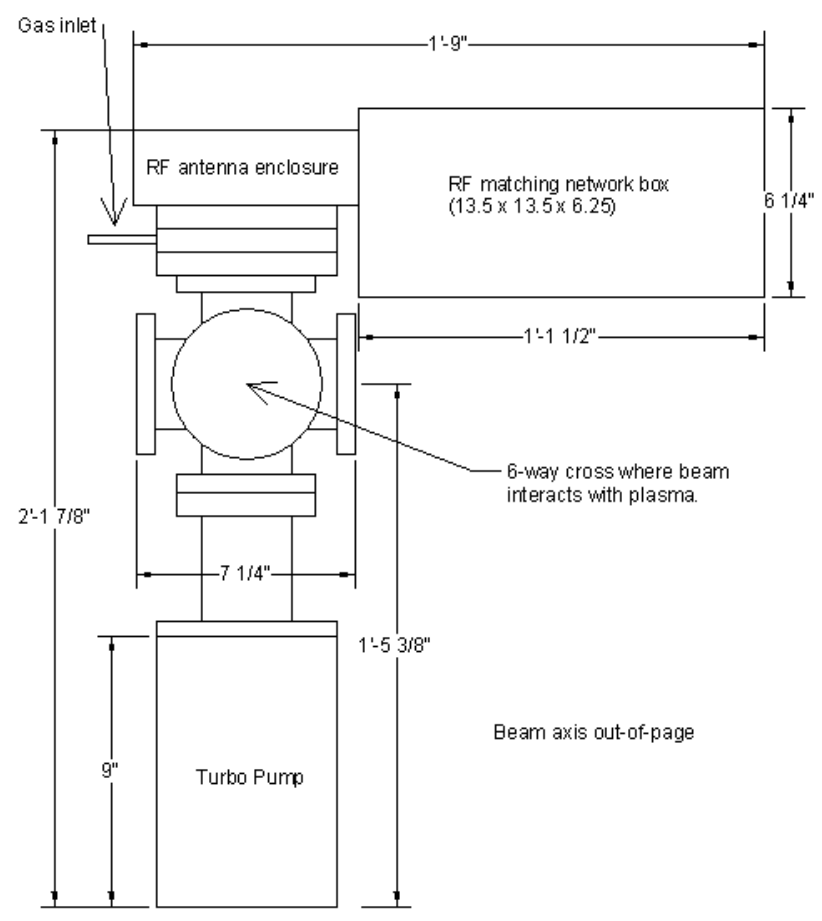

Figure 1: Schematic of pulsed plasma source.

\section{PULSED PLASMA DIAGNOSTICS}

A typical ion gauge controller does not have the time response to measure the quick pressure bursts when a gas puff is applied to the system. However, by measuring the voltage across a resistor placed in series with the ion gauge's collector current, the dynamic absolute pressure in the plasma source can be ascertained. The dynamic pressure measurement is calibrated at a fixed pressure while the pressure gauge is read, and the voltage across the resistor is recorded. To insure that the observed rise time is indicative of the rising gas pressure and not the $R C$ time response of the circuit, the pressure measurement for a given plasma condition was repeated with two different resistors with an order-of-magnitude difference in resistance. The pressure evolutions for the two resistors were in agreement. Applying a $5 \mathrm{~ms}$ gas puff to the plasma source, the pressure sharply rises to $2 \mathrm{mT}$ Torr and pumps away in 1 second. This is expected to be compatible with NTX because it produces $100 \square \mathrm{s}$ ion beams every 15 seconds.

The plasma electron density was measured with a Smart Soft Langmuir probe. It compensates for measurements in RF fields and can make high timeresolved measurements with reproducible plasmas. The pulsed plasma source was observed to be very reproducible in parameters and breakdown time. Furthermore, the Langmuir probe is mechanically stepped through the plasma source for spatial measurements.

\section{PULSED PLASMA SOURCE OPERATION}

To operate the plasma source, the gas valve and the $\mathrm{RF}$ power are triggered at the same time $(t=0)$. In this mode it is easy to deliver more than $2 \mathrm{~kW}$ of RF power to the source, compared to only $1 \mathrm{~kW}$ for continuous plasma source operation. The power is measured with a directional coupler and calibrated diode. The source characteristics for a net forward power of $\sim 3.5 \mathrm{~kW}$ versus time are shown in Fig. 2. Before $t=3.75 \mathrm{~ms}$, the plasma density is less than the sensitivity of the Langmuir probe $\left(\sim 10^{7} \mathrm{~cm}^{-3}\right)$, and the neutral pressure is below the sensitivity of the dynamic pressure measurement $\left(10^{-6}\right.$ Torr). The onset of breakdown is clearly observed in the forward and reflected RF power versus time. The RF matching network is adjusted so that there is a maximum net power delivered to the plasma. At $\mathrm{t}=3.75 \mathrm{~ms}$ the electron density is $10^{11} \mathrm{~cm}^{-3}$ and simultaneous low neutral pressure. The ionization fraction for $\mathrm{t}=3.75-4 \mathrm{~ms}$ is in the range of $50-100 \%$. At later times the power density is not sufficient to sustain the ionization fraction, and the neutral density rises faster than the electron density.

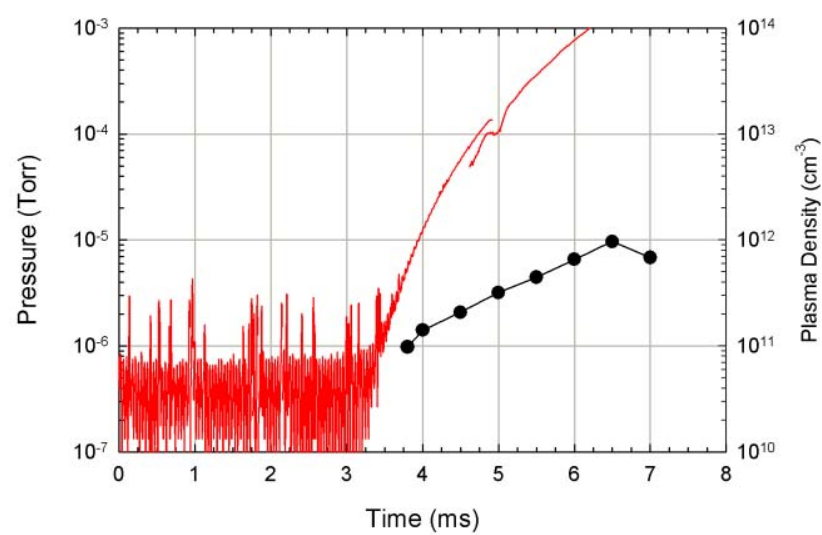

Figure 2: Time evolution of neutral gas pressure and plasma electron density (solid circles) in the pulsed plasma source.

One upgrade under consideration is to replace the gas valve with a faster one to reduce the length of the gas puff and the total amount of gas into the system. Another upgrade would be to increase the RF power level with a pulsed RF supply.

\section{MEASUREMENTS AT LBNL}

The pulsed plasma source was shipped to Lawrence Berkeley Laboratory (LBNL) and set up for initial testing. One difference between the source tests at Princeton and those at LBNL was that the RF generator was not able to provide $3.5 \mathrm{~kW}$ of power at LBNL and was limited to 2.5 $\mathrm{kW}$. This limited the maximum plasma density achieved. 
A number of density measurements were made in the 6way cross with the Langmuir probe. A profile measurement was made transverse to the axis of the plasma source and turbo-pump (Fig. 3). The density is peaked on axis. There is a factor of 2 drop near the plasma source wall radius of $5 \mathrm{~cm}$. Distances greater than $5 \mathrm{~cm}$ from the center of the cross is hidden from the straight-line path of the plasma out of the source. There the density decreases by an order-of-magnitude at a distance $5 \mathrm{~cm}$ from the wall radius of the plasma source.

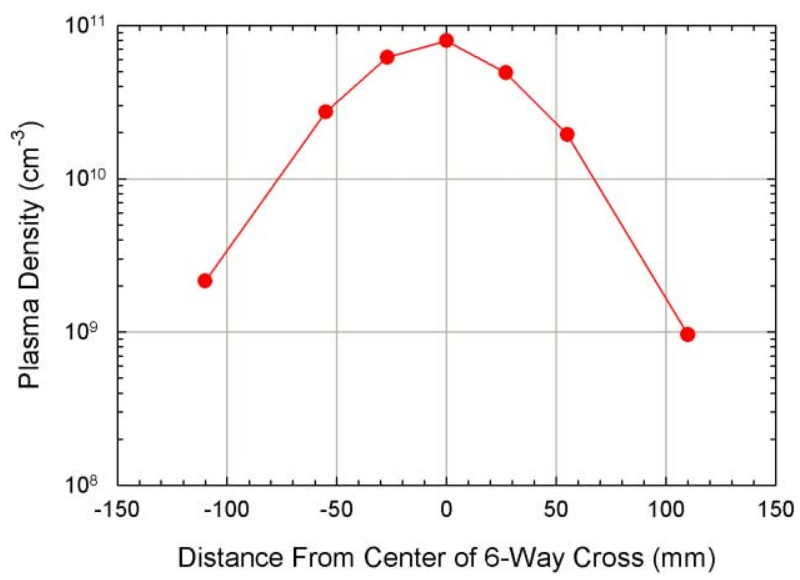

Figure 3: Plasma density profile in the cross transverse to the plasma source.

Another profile measurement was made in the 6-way cross, but along the axis of the plasma source. In this direction the plasma has a steep gradient away from the antenna because of the short plasma skin depth $(\sim 1 \mathrm{~cm})$. This has also been seen in our previous steady-state ECR plasma sources. Consequently, the plasma density drops by 2 orders-of-magnitude across the cross diameter in this direction. In the ECR source, a weak magnetic field was used to reduce this, but is not used in the pulsed source.

Lastly, a density measurement at the center of the cross as a function of RF power was completed (Fig. 4). The electron density increases exponentially as a function of power. The data point near $3.5 \mathrm{~kW}$ was obtained at Princeton, but not at LBNL. Consequently, the source is limited to $5 \times 10^{10} \mathrm{~cm}^{-3}$ density for the NTX experiments until the RF generator can produce the higher power levels.
The pulsed plasma source has been mounted on NTX and neutralization experiments have begun.

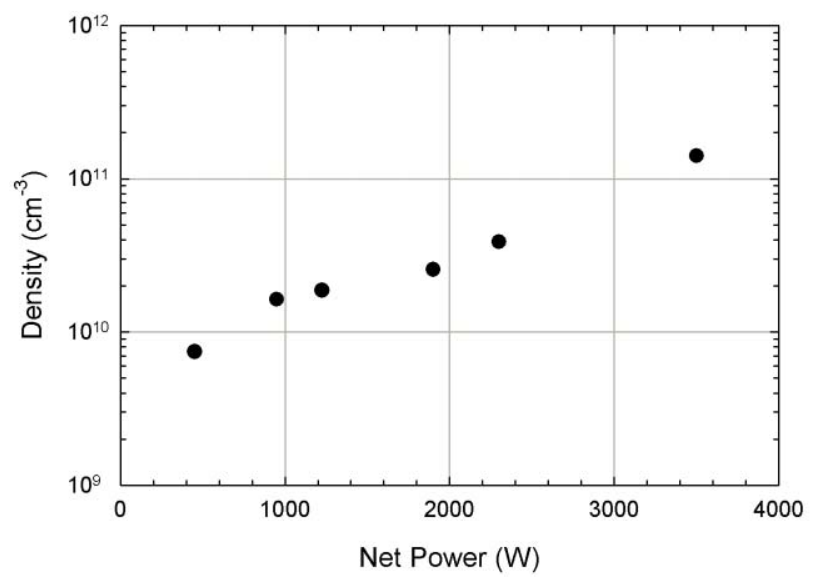

Figure 4: Electron density in the center of the cross as a function of RF power.

\section{FUTURE PLANS}

The RF generator will be brought back to its $4 \mathrm{~kW}$ power capability in order to achieve the densities observed at Princeton. Furthermore, the gas valve will be replaced with a faster one to limit the gas introduced to the NTX vacuum system. Lastly, modified versions of the pulsed plasma source may be examined at Princeton to achieve larger plasmas along the ion beam path. This research was supported by DOE Contract No. DE-AC0276-CH-03073.

\section{REFERENCE}

[1] B. Badger, et al. (1984), "HIBALL-II, An Improved Conceptual Heavy Ion Beam Driven Fusion Reactor Study", KfK-3480, Kernforschungszentrum Karlsruhe Report.

[2] S. Yu, et al., Bull. of Am. Phys. Soc. 46, 195 (2001).

[3] B. G. Logan and D. A. Callahan, Nucl. Instr. and Meth. In Phys. A 415, 468 (1998).

[4] P. C. Efthimion, et al., Laser and Particle Beams 21, 1 (2003). 